\title{
Implantação de um ambulatório de média complexidade no Vale do Taquari - RS: um relato de experiência
} Implementation of a medium complexity ambulatory in Taquari Region, Brazil: an experience report

\author{
Luis Felipe Pissaia, ${ }^{1}$ Carmem Elisa Beschorner ${ }^{1}$ \\ 'Centro Universitário Univates, Lajeado, RS, Brasil.
}

Recebido em: 21/08/2016 / Aceito em: 27/09/2016 / Publicado em: 18/10/2016 Ipissaia@univates.br

\section{RESUMO}

O aumento constante das Doenças Crônicas não Transmissíveis gera uma crescente busca por serviços especializados de atenção à saúde. Uma estratégia a ser explorada é a relação ensino e serviço, focada na resolutividade dos processos saúde e doença, impactando para formação de profissionais generalistas e de comunidade, a um custo financeiro menor. Objetivo: neste contexto, o objetivo é compartilhar vivências referentes a implantação de um ambulatório de média complexidade que está explorando e fortalecendo a relação ensino e serviço no Vale do Taquari/RS. Método: trata-se de um relato de experiência referente ao primeiro semestre de atividades do Ambulatório de Especialidades Médicas do Centro Clínico Univates (AEM), sendo apresentados números de atendimentos registrados em prontuário eletrônico e as vivências in locu. Resultados: durante o período foram realizadas 1.130 consultas e 1.634 diferentes exames, referente a usuários de 25 municípios da região do Vale do Taquari. O serviço ainda não possui contrato com o Estado, sendo o maior número de usuários provenientes do único município que possui contrato estabelecido com o serviço. O acesso para os demais municípios é disponibilizado através do Consórcio Intermunicipal de Saúde (CONSISA), o que inibi os encaminhamentos, devido aos custos. Considerações finais: apesar da dificuldade para a contratualização do serviço junto ao Estado, como referência regional, a experiência proporcionou a problematização da relação ensino e serviço, confirmando esta como uma estratégia de sucesso para qualificação da atenção à saúde, apoio à regionalização da saúde, contribuindo para formação generalista e integral, através de profissionais e estruturas qualificadas, resolutivas, adequadas às demandas locorregionais.

Palavras-chave: Assistência Integral à Saúde; Regionalização; Serviços de Saúde; Recursos Humanos em Saúde.

\section{ABSTRACT}

The steady increase in non-communicable diseases generates a growing demand for specialized health care services. One strategy to be explored is the relationship teaching and service focused on solving processes of health and disease, impacting on general practitioners and community training, at a lower financial cost. Objective: in this context, the objective is to share experiences regarding the implementation of a medium complexity clinic that is exploring and strengthening the teaching and service relationship in Taquari region, RS, Brazil. Method: this is an experience report about the activities of the first semester of Medical Specialties Clinic of the Clinical Center Univates, which presents medical care numbers recorded in electronic medical records and in locu experiences. Results: during the period were carried out 1,130 medical appointments and 1,634 different tests, relating to users of the 25 municipalities of the Taquari region. The service still does not have a contract with the State, being the largest number of users from the only municipality that has a stablished contract with the service. The access to the other municipalities is available through the Intermunicipal Consortium of Health (CONSISA), which inhibits referrals due to costs. Closing Remarks: Despite the difficulty of the legalization of service by the state, as a regional reference, the experience provided the questioning of the teaching and service relationship, 
confirming this as a successful strategy of health care qualification, support for the health regionalization and contributing to general and complete training, through professional and qualified structures, solving, the appropriate regional demands.

Keywords: Comprehensive Health Care; Regional Health Planning; Health Services; Health Manpower.

\section{INTRODUÇÃO}

O Vale do Taquari está localizado na encosta superior do nordeste do Rio Grande do Sul, cerca de 150 quilômetros da capital do Estado, Porto Alegre e segundo a contagem populacional de 2010, a região possui cerca de 320 mil habitantes, sendo em sua maioria descendentes de imigrantes italianos, alemães e açorianos. ${ }^{1} \mathrm{~A}$ região possui tradição na produção agrícola que, aliada ao desenvolvimento dos setores de comércio, de indústrias e de serviços, modificou seu perfil populacional, destacando-se o aumento da expectativa de vida, que atualmente atinge 73,6 anos. ${ }^{2,1}$

Neste sentido, com o gradativo aumento da expectativa de vida, a Confederação Nacional de Municípios (CNM) divulgou em 2011 dados que revelaram um número de 39.117 idosos residentes no Vale do Taquari, caracterizando aproximadamente $12,47 \%$ do contingente populacional. ${ }^{3}$ Os processos de envelhecimento humano englobam fatores psíquicos, sociais e biológicos, que independentemente da idade, compreendem fatores progressivos e irreversíveis propiciando, principalmente, o surgimento de Doenças Crônicas Não Transmissíveis (DCNT). ${ }^{4}$

As DCNT englobam diversas patologias de etiologia diversificada que se caracterizam por influenciar o desenvolvimento de agravos oriundos de doenças como Diabetes Mellitus (DM), Hipertensão Arterial Sistêmica (HAS) e Obesidade ${ }^{5}$. As doenças cardiovasculares são as principais responsáveis por impactos negativos à saúde coletiva, contribuindo significativamente para o aumento dos custos assistenciais, a piora da qualidade de vida, o agravamento das iniquidades e o aumento da pobreza. ${ }^{6-9}$

A partir desta demanda crescente pela qualificação da assistência prestada à população, torna-se necessário a implantação de serviços que contemplem as diretrizes do Sistema Único de Saúde (SUS). Podemos descrever a saúde como um desfecho multifatorial, influenciada pelos modos de organização da produção do trabalho e da sociedade em determinado contexto, evidenciando a influência dos determinantes e condicionantes sociais de saúde, justificando a descentralização da atenção à saúde, fomentando a regionalização da saúde adequando-as às necessidades locorregionais e a aproximação com a Atenção Primária à Saúde (APS). ${ }^{10-12}$

Os serviços de média complexidade visam a assistência à indivíduos que necessitam de atendimentos especializados, sendo integrada à APS, através de um sistema de regulações, necessitando do apoio de recursos tecnológicos e de equipe multidisciplinar capacitada para as atividades. ${ }^{13}$ Nestes espaços de atenção ambulatorial são desenvolvidas inúmeras atividades que contemplam as necessidades da população correspondente a sua área adstrita, sendo incorporada a atenção especializada a oferta de ações de promoção da saúde, prevenção de agravos, tratamento e reabilitação de diversas patologias. ${ }^{14}$ Uma estratégia a ser explorada nesta modalidade de atenção à saúde é a relação ensino e serviço, focada na resolutividade dos processos de saúde e doença e consequentemente na formação generalista e comunitária de seus profissionais e discentes. ${ }^{15}$

Dessa forma, pretende-se compartilhar vivências da implantação de um ambulatório de média complexidade, que vem explorando e fortalecendo a relação ensino e serviço, no Vale do Taquari/RS.

\section{MÉTODO}

Trata-se de um relato de experiência, descritivo e exploratório, em que foram observados os números de atendimentos, exames realizados e a origem dos usuários que frequentaram o primeiro semestre de atividades do Ambulatório de Especialidades Médicas, integrante do Centro Clínico Univates, o qual se localiza em Lajeado/RS, denominada cidade polo do Vale do Taquari.

Os dados apresentados são referentes ao período de 07 de março a 30 de junho de 2016, provenientes de demandas geradas de atendimentos multiprofissionais e de exames diagnósticos, realizados sempre na segunda-feira, nos turnos da manhã e da tarde e na terça-feira, pela manhã, sendo todas as informações registradas em prontuário eletrônico (software Tasy ${ }^{\circledR}$ ), as quais foram disponibilizados pela administração do serviço e incorporados às demais vivências in loco dos profissionais. Foram observados os aspectos éticos, previstos na Resolução $n^{\circ} 466$, de 12 de dezembro de 2012, do Conselho Nacional de Saúde (CNS), tendo como mérito a ênfase com os compromissos éticos dos sujeitos e serviços de saúde, a qual dispensa a necessidade de aprovação pelo Comitê de Ética em Pesquisa (COEP) para este tipo de estudo.

\section{RESULTADOS}

Lajeado é considerada a cidade referência da região do Vale do Taquari, que de acordo com o último censo demográfico, possui 71.445 habitantes, sendo destes 71.180 residentes na zona urbana e 265 residentes na zona rural, caracterizando um alto e crescente grau de urbanização, significando $99,9 \%$ do total populacional. ${ }^{1}$ O ensino superior está presente no município desde 1969 e em 1974 é criada a Fundação Alto Taquari de Ensino Superior, atual Centro Universitário Univates, a qual desde sua criação até a atualidade possui comprometimento e preocupação com o desenvolvimento regional, sendo que em 2015 possuía em torno de 9.197 alunos matriculados.

O Centro Universitário Univates possui uma longa caminhada com diversos cursos superiores na área da saúde, como Biomedicina, Ciências Biológicas (licenciatura e bacharelado), Educação Física (licenciatura e bacharelado), Enfermagem, Estética e Cosmética, Farmácia, Fisioterapia, Gastronomia, Nutrição e Psicologia. Em 2013 também obteve autorização para ofertar o curso de medicina, e em 2014 o curso de Odontologia, o que impulsionou ainda mais investimentos e novas parcerias para ampliação de campos de práticas e es- 
tágios e o fortalecimento da relação ensino e serviço, auxiliando para a qualificação da rede de atenção à saúde locorregional e o fortalecimento do SUS.

Diante deste cenário, identificaram-se municípios com uma APS organizada e uma região com hospitais qualificados, entretanto dificuldades para situações que requerem encaminhamento para atenção especializada e apoio diagnóstico. Nestes casos recorre-se aos grandes centros metropolitanos e a capital, os quais sediam serviços de referência para o SUS, deparando-se a longos períodos de espera (meses, até anos), impactando negativamente para a resolutividade da atenção à saúde regional.

A partir da necessidade de campos de prática e estágios em nível de cuidados de média complexidade, associado a realidade regional, estabeleceu-se a necessidade da criação de um serviço que auxilie o atendimento das demandas locoregionais e proporcione campo de ensino para os futuros profissionais de saúde da região. Atendendo estas necessidades, implantou-se um estabelecimento ambulatorial de média complexidade, denominado Ambulatório de Especialidades Médicas (AEM), integrante do Centro Clínico Univates, destinado a desenvolver uma assistência especializada, eletiva e resolutiva para a população do Vale do Taquari, através de ações que contemplem a clínica ampliada e a interdisciplinaridade.

O Centro Universitário Univates, já possuía alguns serviços de saúde em funcionamento, dentre eles: Clínica-Escola de Fisioterapia, Clínica Universitária de Educação em Saúde (CURES), Ambulatório de Nutrição, Laboratório de Análises Clínicas, Laboratório de Fisiologia do Exercício, Farmácia-Escola, os quais se encontravam implantados por meio dos cursos de graduação da área da saúde e parcerias com as secretarias de saúde regionais, no entanto desenvolviam atividades isoladas. Já, o AEM foi inaugurado em março de 2016, quando também é constituído o Centro Clínico Univates, que visa a integração de todos estes serviços, buscando promover a interação entre os mesmos, a qualificação da assistência e da gestão e assim fortalecer a rede de atenção regional de saúde.

A proposta do AEM é de um serviço escola, que foi concebido através de parcerias articuladas entre o Centro Universitário Univates e Secretarias Municipais de Saúde da região em conjunto com a $16^{\mathrm{a}}$ Coordenadoria Regional de Saúde (CRS), possuindo uma proposta de Contrato de Metas junto ao Estado do Rio Grande do Sul, desde 2015, mas ainda sem contratualização.

O serviço visa atender uma grande parcela da média complexidade oriunda da região, com foco em atendimentos especializados eletivos, exames diagnósticos, ações interdisciplinares de atenção à saúde, proporcionar o desenvolvimento acadêmico de profissionais da área da saúde, aliando as atividades de ensino, pesquisa e extensão no contexto institucional e comunitário. Os atendimentos ficam condicionados à infraestrutura do local, as parcerias e demais convênios firmados, além das necessidades educacionais que permeiam a assistência, possuindo integração entre a APS e demais serviços de média e alta complexidade.

O projeto do AEM prevê a implantação de 16 especialidades médicas, serviços de diagnóstico de apoio e demais multiprofissionais, até 2019, o qual está sendo implantado gradualmente, pois está vinculado ao avanço dos módulos do curso de medicina, iniciando o primeiro semestre com as áreas de cardiologia, pneumologia e hematologia. Atuaram, in loco, profissionais médicos, enfermeiros, fisioterapeutas, farmacêuticos, biomédicos, biólogos, assistentes sociais, administradores, técnicos de enfermagem, técnicos administrativos, técnicos de informação e estagiários, os quais contaram com apoio de nutricionista e psicóloga de outros serviços do Centro Clínico Univates. O número de consultas realizadas por especialidade atuando no AEM, totalizou em 1.130, durante os meses destacados (Tabela 1).

Tabela 1 - Número de consultas realizadas por especialidade.

\begin{tabular}{lc}
\hline ESPECIALIDADES & $\mathbf{N}^{\mathbf{0}}$ \\
\hline Cardiologia & 530 \\
Hematologia & 232 \\
Pneumologia & 349 \\
Enfermagem & 9 \\
Farmácia & 10 \\
\hline TOTAL & 1130 \\
\hline
\end{tabular}

Além das consultas multiprofissionais, o serviço contou com exames diagnósticos de Eletrocardiograma (ECG), Eletrocardiograma de Esforço, Holter, Monitorização de Pressão Arterial Ambulatorial (MAPA), Espirometria, Raio X $(R X)$ e Análises Clínicas, os quais serão ampliados no próximo semestre, com o início de mais três especialidades. Durante o período vigente do estudo realizaram-se 1.634 exames (Tabela 2).

Tabela 2 - Número de exames realizados no AEM.

\begin{tabular}{lc}
\hline EXAMES & $\mathbf{N}^{\mathbf{0}}$ \\
\hline Análises Clínicas & 1236 \\
Eletrocardiograma & 203 \\
Espirometria & 83 \\
Holter & 29 \\
MAPA & 22 \\
Eletrocardiograma de esforço & 61 \\
RX & 9 \\
\hline TOTAL & $\mathbf{1 6 3 4}$ \\
\hline
\end{tabular}

Os eixos de atendimentos realizados pelos serviços incorporam a prática interdisciplinar especializada na prestação de uma assistência qualificada, possuindo dentre seus princípios a autossustentabilidade financeira. A gestão inter-relaciona-se com o ensino acadêmico, responsabilidade em propiciar a resolutividade e reconhecer as limitações e vulnerabilidades de seus processos de trabalhos e ações realizadas.

$O$ início das atividades do AEM foi condicionado à implantação de prontuário eletrônico e sistema de gestão de saúde (TASY ${ }^{\circledR}$ ), o qual é certificado para assinatura eletrônica digital, proporcionado aplicação da metodologia paperless, ou seja, o processo de trabalho é realizado com o auxílio de soluções e sistemas informatizados capazes de substituir integralmente ou parcialmente a utilização do papel, exigindo da equipe a utilização $100 \%$ do prontuário eletrônico e a utilização da assinatura digital eletrônica, o que requer um investimento 
financeiro considerável e uma nova adequação e adaptação dos profissionais de saúde a este padrão de trabalho.

Para este primeiro período de funcionamento, o AEM, não pode contar com recursos financeiros do Estado, visto não ter sido credenciado como uma nova referência para média complexidade. Este fato levou à busca por alternativas financeiras que auxiliassem a custear uma parcela dos custos dos serviços, conforme pode ser verificado nos atendimentos prestados por convênios em cada município que aderiu a proposta (Tabela 3).

Tabela 3 - Municípios que utilizaram o serviço x Formas de convênio

\begin{tabular}{|c|c|c|c|}
\hline MUNICÍPIOS & CONSISA & sus & Gratuidades \\
\hline Arroio do Meio & 44 & & 16 \\
\hline Bom Retiro do Sul & 2 & & 40 \\
\hline Boqueirão do Leão & & & 8 \\
\hline Canudos do Vale & 4 & & \\
\hline Capitão & & & 9 \\
\hline Cruzeiro do Sul & & & 36 \\
\hline Doutor Ricardo & 9 & & \\
\hline Estrela & 78 & & 208 \\
\hline Fazenda Vila Nova & 1 & & \\
\hline Forquetinha & 6 & & \\
\hline Imigrante & 2 & & 6 \\
\hline Lajeado & 2 & 1782 & 58 \\
\hline Marques de Souza & & & 43 \\
\hline Paverama & 2 & & 12 \\
\hline Poço das Antas & & & 17 \\
\hline Pouso Novo & 9 & & 12 \\
\hline Relvado & 11 & & 1 \\
\hline Santa Clara do Sul & 1 & & 19 \\
\hline São José do Herval & 1 & & \\
\hline São Valentim do Sul & 1 & & \\
\hline Sério & 18 & & 9 \\
\hline Taquari & & & 20 \\
\hline Teutônia & 12 & & 59 \\
\hline Travesseiro & & & 13 \\
\hline Westfália & 4 & & \\
\hline TOTAL: & 207 & 1782 & 586 \\
\hline
\end{tabular}

O objetivo é cadastrar o serviço junto ao Estado como referência regional, porém como isto ainda não aconteceu, algumas alternativas foram instituídas para auxiliar no financiamento do serviço. O município sede do AEM contratou parcela da sua capacidade de atendimento, através de um contrato direto. Outra alternativa encontrada foi o credenciamento junto ao Consórcio Intermunicipal de Saúde do Vale do Rio Taquari (CONSISA VRT), que é composto por vários municípios da região, para pleitear recursos e serviços específicos. Os municípios associados, conforme suas necessidades, compram esses serviços de qualidade e a preços compatíveis, com base nos preços da tabela SUS, viabilizando condições mais plenas de saúde aos seus munícipes. Este convênio disponibilizou o acesso aos demais municípios do vale, entretanto sem grande adesão, pois acaba onerando os custos com a saúde para estes, o que muitas vezes não compete aos mesmos.

Além destas dificuldades, também existia a necessidade de usuários frequentando o serviço, pois os atendimentos estavam associados à aulas práticas que estavam programadas para este espaço, o que levou o serviço a disponibilizar, temporariamente, vagas de gratuidade de atendimento e assim viabilizar o ensino.

\section{DISCUSSÃO}

A implantação de instituições universitárias em determinadas regiões demográficas, causam impactos a médio e longo prazo; neste caso, uma entidade comunitária maximiza seu compromisso com a população local. As ofertas de serviços, tanto acadêmicos, quanto comunitários são planejados e implementados em conjunto, através de recorrentes problematizações da realidade social.

Os principais objetivos destas instituições comunitárias de ensino superior é suprir as demandas acadêmicas que emergem na sociedade atual, oferecendo em contrapartida uma formação profissional integral e comprometida com as próprias necessidades em seu meio de convívio. ${ }^{16}$ A comunidade local permanece sendo a maior beneficiaria por abrigar as instancias de serviços que esta instituição oferece e acolher os futuros profissionais, que vinculados ao meio acadêmico contextualizam suas práticas na região. ${ }^{17}$

Outra situação que merece destaque, discorre sobre as instituições de ensino superior que oferecem cursos na área da saúde, devido ao seu papel, enquanto formador de profissionais, tanto capacitados teoricamente, quando atuantes na realidade regional. Este compromisso firma-se através da implantação e implementação de serviços, em sua maioria com parcerias locais que visam incentivar as vivências acadêmicas e ao mesmo tempo auxiliar as demandas sociais da região.

Os cursos da área da saúde, com ênfase para o de medicina possuem dificuldades quanto ao número suficiente de campos de práticas nas regiões onde as instituições de ensino estão instaladas, o que influencia diretamente na formação generalista de profissionais da saúde, preconizada pelo Ministério da Educação (MEC). ${ }^{18}$ Uma das alternativas encontradas pelas escolas de saúde em todo o mundo é a implantação de serviços que comtemplem a demanda acadêmica, visando também parcerias com entidades locais que estimulem seu planejamento e incorporação à rede de apoio. ${ }^{19}$

Neste contexto, encontram-se dificuldades em atrelar a prática da interdisciplinaridade frente aos novos serviços implantados, ao passo que, os antigos modelos vigentes preconizam uma prática setorializada e marcada pela atuação isolada de seus profissionais. Através da incorporação de novos processos de trabaIho e teorias de atuação profissional, as instituições de universitárias iniciaram uma transformação no ensino ao incentivar a prática interdisciplinar de seus estudantes, e implementar estes modelos em seus próprios meios de atuação. ${ }^{18}$

Conforme observado, encontraram-se dificuldades na viabilidade de investimentos para o acesso ao serviço pelos municípios, o que é evidenciado ao observar a demanda alavancada durante o período de gratuidade e a queda acentuada logo após; no entanto, compreendem-se as dificuldades financeiras pela qual os municípios estão passando. A falta de investimento e repasse de financiamentos por parte do SUS prejudica 
o andamento de projetos de apoio a atual infraestrutura dos municípios, nestes casos as demandas por especialidades ficam reprimidas a filas de espera nos grandes centros de saúde. ${ }^{20}$

Perpassando por outros sentidos, a incorporação do serviço à realidade regional flexibiliza as demandas por atendimentos especializados e reafirma o compromisso com o ensino dos futuros profissionais da área da saúde, induzindo através de boas práticas acadêmicas e o uso de tecnologias de apoio a excelência nos atendimentos prestados. Um serviço de saúde com foco no ensino e na assistência, ao ser implantado em um contexto pré-definido encontra várias dificuldades, entre elas a de conciliar ambas as atividades, subsidiando sua sustentabilidade e agregando valor, à medida que seu desenvolvimento seja percebido pelas demais iniciativas públicas e privadas. ${ }^{21}$

\section{CONSIDERACÕEES FINAIS}

A partir da análise dos resultados verificou-se que as vivências incorporadas à implantação do AEM são condizentes com seu planejamento inicial e seguem o limiar preconizado. As atividades em andamento seguem a contextualização e problematização do ensino e assistência, que embora apresentem algumas dificuldades iniciais na contratualização de parcerias, os benefícios superam as dificuldades e oferecem a ambos uma estrutura capacitada e eficiente.

Além de oferecer à comunidade um serviço diferenciado, o Centro Universitário Univates possui o compromisso em auxiliar nas demandas regionais, o que é previsto em seus princípios. A incorporação do projeto do Centro Clínico Univates ao eixo de apoio regional alavanca a potencialidade da instituição enquanto ferramenta de modificação e quebra de paradigmas, com ênfase no trabalho interdisciplinar, instrumento eficaz de intervenção comunitária.

Para tanto, a integração entre o ambiente universitário e comunidade torna-se efetivo e colaborativo de boas práticas sociais, ao passo que, o limiar entre ensino e assistência assume uma postura de motivação e flexibilização dos laços entre indivíduos e serviços de saúde, o que é perceptível através dos retornos positivos fornecidos, tanto pelos usuários, quanto pelos municípios que acessaram o serviço. Apesar do cenário econômico desfavorável para novos investimentos em saúde em todo o país, o AEM segue com o processo de contratualização junto ao Estado, contando com o apoio da região e assim poder concretizar e fortalecer o desejo pela regionalização da saúde e um espaço de construção de um ensino qualificado.

\section{REFERÊNCIAS}

1. Instituto Brasileiro de Geografia e Estatística. Síntese de Indicadores Sociais: Uma Análise das Condições de Vida da População Brasileira, 2010. Disponível em: http:// www.ibge.gov.br/home/presidencia/noticias/imprensa/ ppts/0000000144.pdf. Acesso em: 23 jul. 2016.

2. Costa AEK, Ferla NJ, Backes GA, Moreschi C. A equipe multiprofissional e o gerenciamento dos investimentos públicos aplicados na saúde dos idosos. Revista Destaques Acadêmicos, 2015; 7(3).

3. CNM. Confederação Nacional de Municípios. 2011. Disponível em: <http://www.cnm.org.br/dado geral/mumain. asp?ildMun=100143425>. Acesso em: 23 jul. 2016.

4. Portugal BBG, Junior PSF, Bastos LD, Carvalho PL. A longevidade da população e os desafios envolvidos. Revista Fluminense de Extensão Universitária 2016;4(1.2):07-08.

5. Alves MS, Araújo MDCF, Nascimento MP, Ribeiro FC, Rebouças PT, Santos TA, Oliveira JI. Grupo Terapêutico com Idosos Sobre o Autocuidado nas Doenças Crônicas. Journal of Health Sciences 2016;18(1):52-55. doi: 10.17921/2447-8938.2016v18n1p52-55.

6. European Society of Cardiology. European guidelines on cardiovascular disease prevention in clinical practice. European Heart Journal 2003;(24):1601-1610. doi: 10.1016/ S0195-668X(03)00347-6.

7. Brasil. Cadernos de Atenção Básica: n. 14 - prevenção clínica de doença cardiovascular, cerebrovascular e renal crônica do Ministério da Saúde. Brasília: Ministério da Saúde, 2006.

8. SBC. VI Diretriz Brasileira de Hipertensão. Sociedade Brasileira de Cardiologia, 2010;1(95 supl. 1):1-51.

9. Brasil. Ministério da Saúde. Secretaria de Vigilância em Saúde. Departamento de Análise de Situação de Saúde. Plano de ações estratégicas para o enfrentamento das doenças crônicas não transmissíveis (DCNT) no Brasil 2011-2022 / Ministério da Saúde. Secretaria de Vigilância em Saúde. Departamento de Análise de Situação de Saúde. Brasília: Ministério da Saúde, 2011.

10. Santos AMD, Giovanella L. Managing comprehensive care: a case study in a health district in Bahia State, Brazil. Cadernos de Saúde Pública 2016;32(3). doi: 10.1590/0102-311X00172214.

11. Lobato LDVC, Martich E, Pereira I. Elected mayors, health decentralization and commitments with the SUS. Saúde em Debate 2016;40(108):74-85. doi: 10.1590/0103-110420161080006.

12. Brasil. Secretaria de Vigilância em Saúde. Secretaria de Atenção à Saúde. Política Nacional de Promoção da Saúde. 3. ed. Brasília: Ministério da Saúde, 2010.

13. Alves DA, Kuroishi RCS, Mandrá PP. Electronic medical records: perceptions from speech-language-hearing pathology undergraduate students and professionals. Revista CEFAC 2016;18(2):385-391. doi: 10.1590/19820216201618217915.

14. Panozzo VM, Costa PRB. Assistência a saúde nos serviços de alta complexidade no Brasil: uma experiência do trabalho do Assistente Social. Revista Ampliar 2016;2(2).

15. Kitamura ES, Bastos RR, Palma PV, Leite ICG. Avaliação da satisfação dos usuários dos Centros de Especialidades Odontológicas da macrorregião Sudeste de Minas Gerais, 2013. Epidemiologia e Serviços de Saúde 2016;25(1):137148. doi: 10.5123/S1679-49742016000100014.

16. Cruz CO, Stoppa CP, Silva DC, Silva JDLL, Maia SF. Bolsa social: quem está inserido?. Multitemas 2016;(32).

17. Cunha LA. The enthronement of religious education in the National Common Curriculum Base. Educação \& Sociedade, 2016;37(134):266-284.

18. Cyrino EG, Cyrino ADPP, Reeves S, Batista NA, Costa MVD, Azeredo GD, Batista SHSDS. Educação interprofissional no Brasil: construindo redes formativas de educação e trabalho em saúde. Interface (Botucatu. Online), 2016. doi: 10.1590/1807-57622015.0700.

19. Baldoino AS, Veras RM. Análise das atividades de 
IMPLANTAÇÃO DE UM AMBULATÓRIO DE MÉDIA COMPLEXIDADE NO VALE DO TAQQUARI/ RS: UM RELATO DE EXPERIÊNCIA

Luis Felipe Pissaia, Carmem Elisa Beschorner

integração ensino-serviço desenvolvidas nos cursos de saúde da Universidade Federal da Bahia. Revista da Escola de Enfermagem da USP 2016;17-24. doi: 10.1590/S0080623420160000300003.

20. Marques RMM, Mendes Á. Os dilemas do financiamento do
SUS no interior da seguridade social. Economia e Sociedade 2016;14(1):159-175.

21. Amorim DP. Análise Financeira de Hospitais: O Caso da Santa Casa de Misericórdia de Itaguara. RAGC 2016;4(11).

Como citar: PISSAIA, Luis Felipe: BESCHORNER, Carmem Elisa. Implantação de um ambulatório de média complexidade no Vale do Taquari/RS: um relato de experiência. Cinergis, Santa Cruz do Sul, v. 17, n. 4, out. 2016. ISSN 2177-4005. Disponivel em: <https://online.unisc.br/seer/index.php/cinergis/article/view/8139>. Acesso em: 11 out. 2016. doi:http://dx.doi. org/10.17058/cinergis.v17i4.8139. 